\title{
Ion Funnels for the Masses: Experiments and Simulations with a Simplified Ion Funnel
}

\author{
Ryan R. Julian, Sarah R. Mabbett, and Martin F. Jarrold \\ Department of Chemistry, Indiana University, Bloomington, Indiana, USA
}

\begin{abstract}
A modified ion funnel is described. Counterintuitively, increased spacing between electrodes results in enhanced "focusing" of the ions through the funnel. Consequently, the internal diameter (i.d.) of the funnel need not decrease to the conductance limit (as in previous designs). A simple dc-only lens, which also serves as the conductance limit, combined with the natural flow of gas is used to extract the ions from the funnel. Ions with mass to charge ratios varying between 75 and $3000 \mathrm{~m} / \mathrm{z}$ are passed through the funnel with no apparent discrimination. The funnel can be operated under mild conditions that preserve weakly bound noncovalent complexes. After testing several designs, a thin closely spaced dc lens was found to be the best solution for extracting ions. A simple method for simulating ion trajectories at nonzero pressures based on ion mobility and explicit diffusion is described. This theoretical approach was used to design and calculate ion trajectories for the modified funnel presented here. Finally, the increased spacing between electrodes in the current funnel significantly relaxes machining constraints, reduces cost, and enhances ease of use versus previous funnel designs. (J Am Soc Mass Spectrom 2005, 16, 1708-1712) (C) 2005 American Society for Mass Spectrometry
\end{abstract}

$\mathrm{E}$ lectrospray ionization (ESI) has enabled the study of a wide range of molecules that were previously inaccessible through routine mass spectrometric techniques [1]. Nevertheless, efficiently transferring ions produced by ESI at atmospheric pressure into the low-pressure environment that is required for mass spectrometry remains a significant challenge. Various source designs have addressed this problem, but recent advances have been made with the introduction of the ion funnel [2a, 2b]. An ion funnel is a stack of closely spaced ring electrodes with decreasing internal diameters (i.d.'s) [3]. A combination of rf and dc potentials is used to "focus" the ions at pressures ranging from $\sim 10$ to 0.1 torr. The rf electric fields are used to confine ions radially, while a dc potential drives them toward the exit of the funnel. Ions exiting a capillary into the funnel are initially free to expand radially with the gas expansion, which naturally occurs as a result of the drop in pressure. After a brief expansion, ions are stopped by radially confining rf potentials and then recompressed by the dc gradient and shrinking ring electrode i.d.'s.

Early reports indicated that ion funnels increase ion transmission by at least an order of magnitude when compared with a capillary skimmer arrangement. However, the initial design was not efficient at collecting ions with low $\mathrm{m} / \mathrm{z}$ ratios. Simulations suggested that this

Published online August 10, 2005

Address reprint requests to Dr. Ryan R. Julian, Department of Chemistry, Indiana University, 800 East Kirkwood Avenue, Bloomington, IN 47405, USA. E-mail: ryjulian@indiana.edu problem could be fixed by decreasing the spacing between the lenses to be less than the diameter of the smallest ring electrode $[4 a, 4 b]$. This arrangement provided better transmission for low $\mathrm{m} / \mathrm{z}$ ions, but it also necessitated the use of zero insertion force socket adapters and custom-printed circuit boards to enable facile attachment of the required electronics [5]. After these initial pioneering efforts by Smith and coworkers, several other groups have implemented similar ion funnels into a variety of instruments [6a, 6b]. Later modifications to the basic design included the use of a jet disrupter to reduce gas pumping loads and the implementation of a multicapillary inlet capable of admitting more ions into the funnel [7]. Recently, a dual channel ion funnel was developed to enable sampling through two distinct capillaries [8].

Despite the tremendous advantages offered by ion funnels, the widespread use of these devices still has not occurred. In the present work, results from both experiments and simulations for a modified ion funnel design are given. Although the spacing between electrodes must be significantly smaller than the i.d. of the smallest ring electrode as shown previously [4a, 4b], it is still possible to design a funnel with large spacing between the electrodes provided that the funnel does not reach the conductance limit. In this alternate configuration, increased spacing results in more efficient ion compression, allowing the ions to be extracted through a dc-only lens, which serves as the conductance limit. In the present work, the spacing between lenses is increased to $5 \mathrm{~mm}$ with an i.d. of $7.9 \mathrm{~mm}$ for the exit ring electrode. This spacing is 2-10 times larger than the 


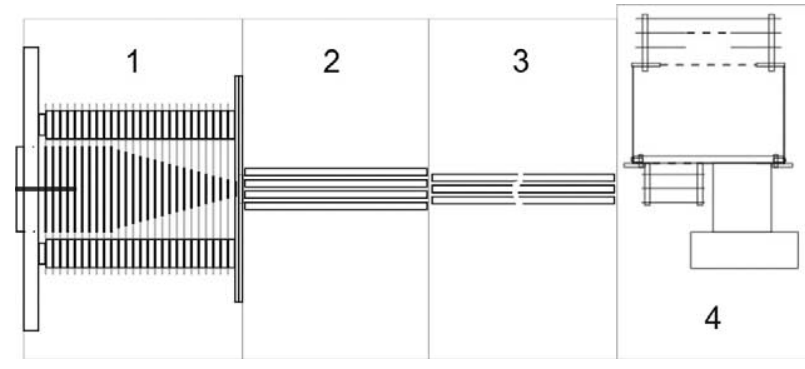

Figure 1. Block diagram of the instrument. Ions are generated by an electrospray source, collected in the ion funnel (1), transferred through an octopole (2) and then hexapole (3), and, finally, analyzed by a TOF-MS (4). Differential pumping regions are labeled 1-4 in accordance with the description in the text.

lens spacing in previous ion funnels, yet there is no observed discrimination between 75 and $3000 \mathrm{~m} / \mathrm{z}$. Additionally, there is sufficient room to accommodate standard resistors and capacitors. The new funnel can be operated under mild conditions that preserve weakly bound noncovalent complexes intact. Alternatively, harsh conditions can be used by modulating the dc potentials to heat or even fragment ions. The experimental results are discussed in relation to a simple simulation model that relies on ion mobility and explicit ion diffusion to predict ion trajectories using fields obtained from SIMION [9]. This appears to be a simple, yet effective, method for modeling ion trajectories at nonzero pressures (greater than $\sim 50$ millitorr).

\section{Experimental Methodology}

\section{Instrument Description}

The general instrument design is shown in Figure 1. Briefly, an ESI source with a heated bath gas configuration generates ions that are then transferred via a $0.5-\mathrm{mm}$ i.d. capillary into an ion funnel in the first differential pumping region. Ions are then passed from the funnel into an octopole ion guide in the second differential pumping region. From there, ions pass into a hexapole ion guide and finally into a time-of-flight mass spectrometer (TOF-MS) in the third and fourth differential pumping regions, respectively. The TOF-MS has not been fully optimized at this point (in terms of resolution), but the performance is sufficient to test the properties of the funnel.

A photo of the entire ion funnel assembly is shown in Figure 2. The ion funnel consists of a 26-electrode stack of $0.51-\mathrm{mm}$-thick plates separated $5.1 \mathrm{~mm}$ by Delrin washers. The first 10 electrodes in the stack are copper with a constant 38.1-mm i.d., and the remaining 16 stainless steel electrodes have an i.d. that begins at $36.3 \mathrm{~mm}$ and reduces linearly to $7.9 \mathrm{~mm}$. A voltage divider is used to provide a linear dc voltage gradient between electrodes 1 and 26 and consists of $1 / 4-W$, $180-\mathrm{k} \Omega$ metal film resistors. The resistors are soldered to beryllium copper spring clips manufactured in-house, which are then attached to each adjacent electrode.
These spring clips are easily fashioned with a small diameter rod template and a pair of pliers. The initial and final dc voltages $(\sim 2 \mathrm{~V} / \mathrm{cm})$ on the funnel can be independently controlled. The dc extraction lens is simply a 1.52-mm-thick stainless steel plate with a 1.8-mm i.d. spaced $2.54 \mathrm{~mm}$ from the last $\mathrm{rf}$ electrode. The rf voltages $(\sim 100-300$ peak-to-peak voltage $[\mathrm{Vp} / \mathrm{p}])$ of equal amplitude but opposite phase are applied to adjacent electrodes by way of $1000-\mathrm{pF}$ capacitors. An rf generator [10] built in-house provides rf voltage to the ion funnel at a frequency of $\sim 650 \mathrm{kHz}$. The funnel is typically operated at a pressure of $\sim 220$ millitorr as measured directly by an MKS 925C MicroPirani gauge (model 925C-41 with RS232 communications; MKS Instruments, Inc., Wilmington, MA), but it has also been used successfully at pressures ranging from several torr to $\sim 50$ millitorr.

The octopole ion guide consists of eight $127-\mathrm{mm}$ length rods with a $3.2-\mathrm{mm}$ diameter. The center of the rods lies on a 12.2-mm diameter and is fixed in place with a polyetherethylketone support. The octopole assembly is mounted onto four stainless steel rods that fit exactly into a stainless steel flange with a conductancelimiting orifice of $2.5 \mathrm{~mm}$ i.d. The hexapole ion guide consists of six $66.7-\mathrm{cm}$-length rods with a diameter of $1.6 \mathrm{~mm}$, and the center of the rods sit on a 9.2-mm diameter. The typical operating $\mathrm{rf}$ voltage for both multipoles is $250 \mathrm{Vp} / \mathrm{p}$ at a frequency of $\sim 1 \mathrm{MHz}$ for the octopole and $\sim 800 \mathrm{kHz}$ for the hexapole. The

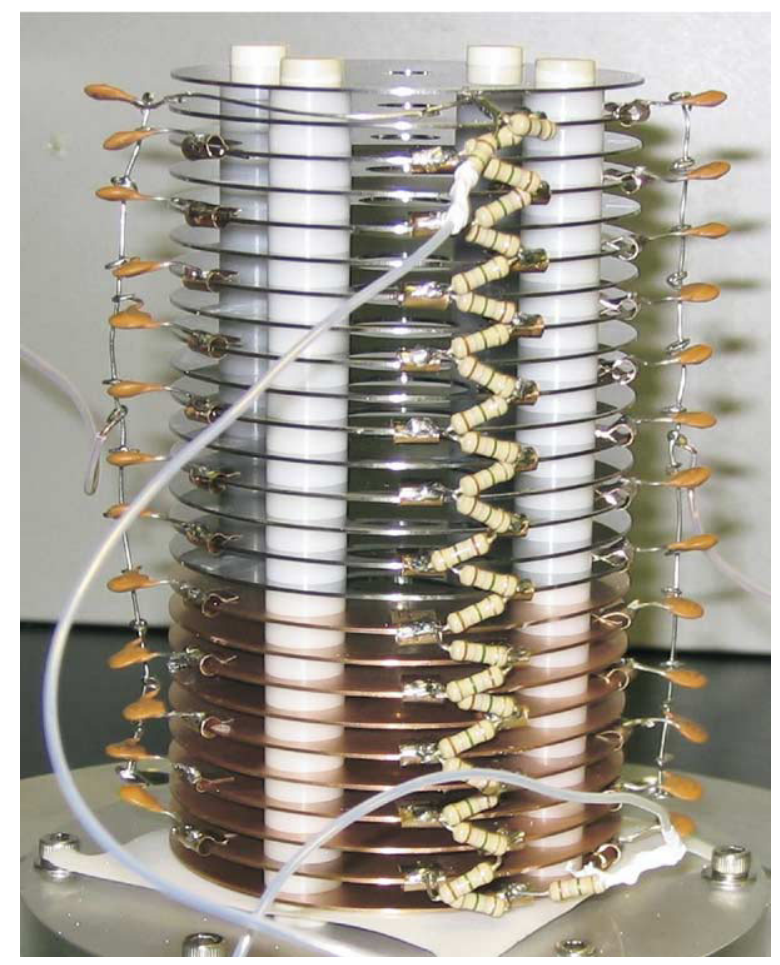

Figure 2. Photo of the actual completed ion funnel assembly. The funnel is $\sim 5 \mathrm{in}$. long and is spring loaded from the bottom. All electrical connections to the lenses of the funnel are made with beryllium copper spring clips, which can be easily removed. 
octopole and hexapole ion guides are in different pressure regions, with the octopole normally at $1 \times 10^{-4}$ torr and the hexapole usually operating at $1 \times 10^{-7}$ torr. The completed TOF-MS will be fully described in a later publication.

\section{Ion Trajectory Simulations}

SIMION was used to build electrode geometries and determine both $\mathrm{rf}$ and dc contributions to the fields, which can be set to any desired value. Ion trajectories were then calculated based on ion mobility and diffusion. This model assumes no initial velocity for ions; instead, ion motion is determined entirely by the instantaneous force from the electric fields at each point in the simulation and the stochastic motion due to diffusion. The presence of a static bath gas is assumed (i.e., the influence of gas flows on ion trajectories is not explicitly treated). The model is only valid at nonzero pressures where thousands of collisions occur between the ion and the bath gas during the course of a trajectory and where field strengths are within the low field limit [11]. Ion trajectories that leave the low field limit can still be calculated but are approximations. Space charge effects are not explicitly accounted for, although other models have done so (4b). Diffusion is modeled by stepping the ion randomly in all three dimensions according to

$$
\mathrm{dx}, \mathrm{y}, \mathrm{z}= \pm(2 \Delta \mathrm{t} D)^{1 / 2}
$$

while simultaneously moving the ion in response to the dc and rf voltages:

$$
\mathrm{dx}, \mathrm{y}, \mathrm{z}=e D / k T \Delta t\left(\mathrm{E}_{\mathrm{dc}}+\mathrm{E}_{\mathrm{rf}}\right)_{x, y, z}
$$

In these equations $D$ is the diffusion constant, $e$ is the ionic charge, $k$ is the Boltzmann constant, $\Delta t$ is the time step, $T$ is the temperature of the gas, and $E_{\mathrm{dc}}$ and $E_{\mathrm{rf}}$ are the $\mathrm{dc}$ and $\mathrm{rf}$ electric fields, respectively [11]. The time step is chosen such that the rf sine wave is sampled sufficiently (typically, $\sim 15$ times) and further reductions lead to no net change in the results.

\section{Results and Discussion}

\section{Theory and Simulation}

The regions of radial ion confinement generated by rf fields within a ring electrode system can be approximated by defining effective field-free and strong field regions. A simple first-order approximation for defining these regions is shown in Figure 3. Simply put, the field contributions from each lens element are canceled out by the two adjacent elements when the distance from the lens is equal to half the distance from the two adjacent lenses. The fields cancel out at this point because the contribution from the center lens is twice as strong as the individual contributions from the two

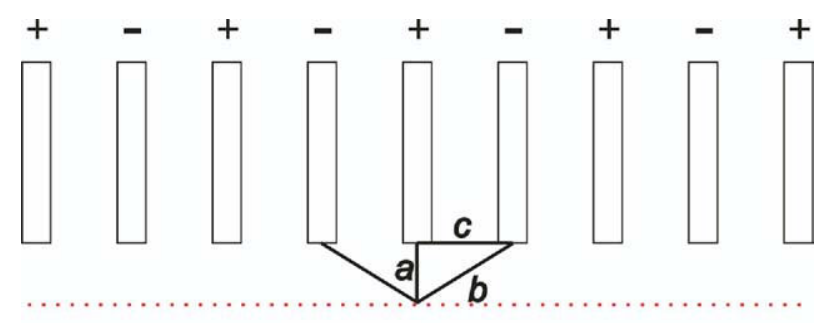

field free region

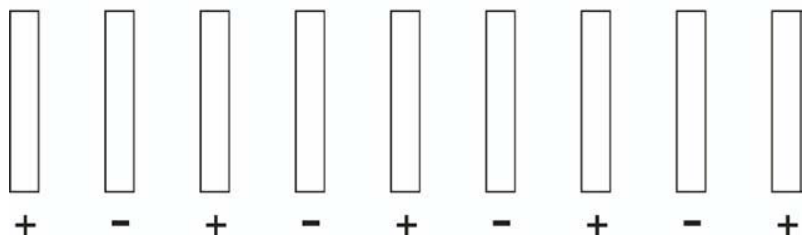

Figure 3. Strong field and field-free regions can be approximated using a simple relationship. An essentially field-free region begins when the distance from an electrode is $60 \%$ of the distance between the electrodes.

adjacent lenses, but there are two of them, leading to net cancellation. This cancellation will occur when eq 3 is satisfied:

$$
a=\left(3^{-0.5}\right) \mathcal{c}
$$

or when the distance away from a lens element is $\sim 60 \%$ of the distance between two lens elements. In other words, increasing the spacing between lenses will lead to enhanced focusing [12] of the ions because the fields are allowed to penetrate further away from each electrode, within certain limitations explained later. The approximate field-free region in which ions can reside is shown by the dashed lines in Figure 3. Second-order and higher effects prevent this region from being truly field free, but these contributions are much smaller in magnitude and do not significantly influence ion trajectories. Inside of the dashed line, field strength increases sharply, with fields rising more quickly as $c$ becomes smaller. Consequently, extreme spacing (very large $c$ ) is not beneficial because of excessive softening of the field strength in the radial direction.

The simple relationship between lens spacing and ion focusing shown in Figure 3 can be very useful in ion funnel design. The results for 50 simulated ion trajectories in our modified ion funnel are shown in Figure 4. The individual trajectories were calculated by the ion mobility/diffusion approach described above. The approximate field-free boundary lines as calculated using eq 3 are shown as dashed lines in Figure 4. The more elaborate simulation confirms that ions that leave the field-free region are quickly redirected away from the lens electrodes. Thus, the effective volume available to the ions can be quickly approximated using eq 3 . The percent transmission through this funnel is $94 \%$ through the dc extraction electrode for 500 simulated 


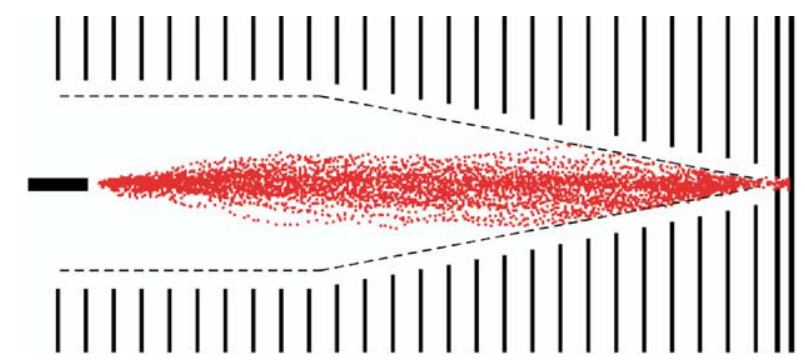

Figure 4. Results for 50 simulated ion trajectories through the modified ion funnel. Ion trajectories remain primarily within the confines of the field-free region as delineated by the dashed lines based on the relationship shown in Figure 3. The calculated transmission efficiency is $94 \%$. The $\mathrm{rf}$ voltage was set to $200 \mathrm{Vp} / \mathrm{p}$ with a dc drop of $30 \mathrm{~V}$ across the funnel. The first 26 lines (from left to right) represent the electrodes of the funnel, having both rf and dc voltages. The last two lines represent the dc extraction region and have no rf voltage.

ion trajectories, although we note that the actual value may be lower due to radial gas flow (at the source) and space charge effects that are not accounted for.

a

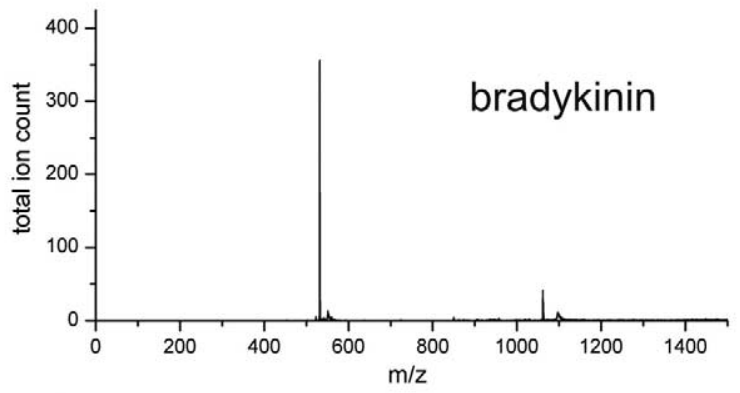

b

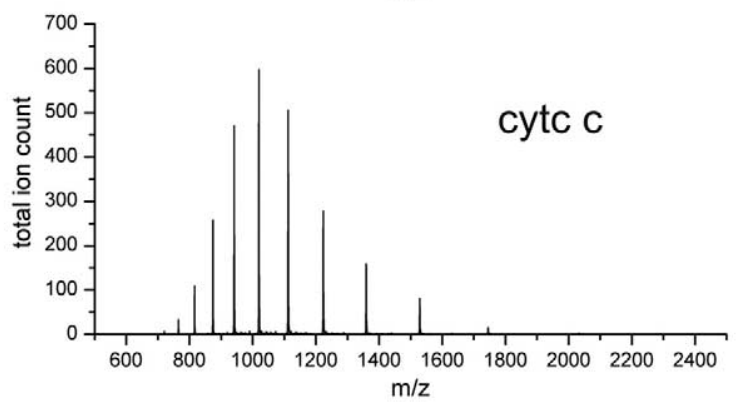

C

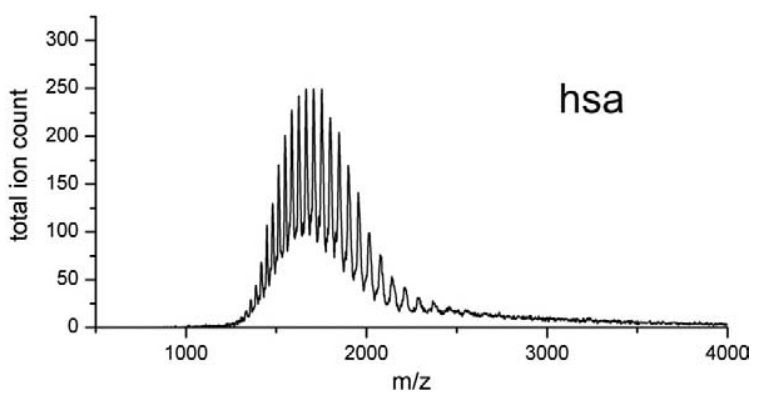

Figure 5. Typical mass spectra observed with several biomolecules ranging in size from a small peptide to a large protein. (a) Spectrum of bradykinin; (b) spectrum of cytochrome $c$; (c) spectrum of human serum albumin.
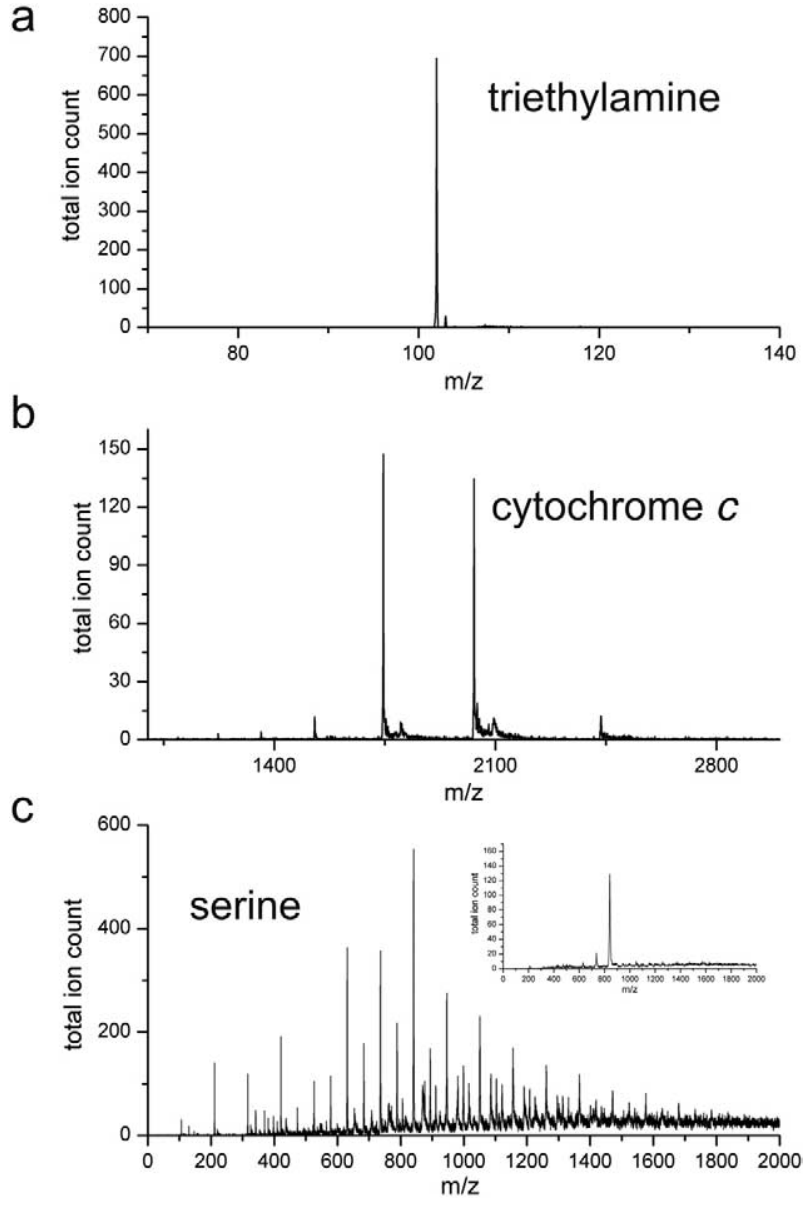

Figure 6. Both high and low $m / z$ ions are transmitted through the funnel. (a) Protonated TEA is transmitted through the funnel in high abundance. Previous data acquired with a quadrupole mass spectrometer demonstrated mass transmission down to $72 \mathrm{~m} / \mathrm{z}$. (b) Cytochrome $c$ sampled from a buffered solution yields high $\mathrm{m} / \mathrm{z}$ peaks corresponding to charge states with four to six protons. (c) Fragile, noncovalently bound serine clusters are easily transmitted through the funnel. The protonated serine octamer becomes even more prominent under milder conditions as shown in the inset.

\section{Preliminary Results}

Typical mass spectra acquired using the ion funnel are shown in Figure 5. The results for bradykinin (average molecular weight, $\sim 1060 \mathrm{Da}$ ), cytochrome $c$ (molecular weight, $\sim 12,000 \mathrm{Da}$ ), and human serum albumin (molecular weight, $\sim 65,000 \mathrm{Da}$ ) are shown in Figure 5a-c, respectively. Biomolecules ranging from small peptides to large proteins are easily sampled with the new funnel. The efficiency of the funnel was tested by comparing the total ion counts obtained for these three systems with the rf voltages turned on and off. When no rf voltage is applied to the funnel, it fails to focus ions in the radial dimension and the total ion count diminishes by a factor $\sim 60$ on average. This indicates that the funnel is operating in the manner predicted by our simulations.

The $m / z$ range of the funnel is illustrated in Figure $6 a, b$. We have observed no discrimination effects for 
ions ranging from $\sim 75$ to $3000 \mathrm{~m} / \mathrm{z}$, which corresponds roughly with the typical range for ESI experiments. In Figure 6a, protonated triethylamine (TEA) is transmitted through the funnel in high abundance. Previous experiments using a large voltage drop from the capillary to the funnel showed transmission of a TEA fragment at $\mathrm{m} / \mathrm{z} 72$ (data not shown). Possibly, ions of smaller $\mathrm{m} / \mathrm{z}$ could also be transmitted, but we have not explicitly tested this possibility. Figure $6 \mathrm{~b}$ shows the results from electrospraying a buffered solution of cytochrome $c$. Several high $\mathrm{m} / \mathrm{z}$ peaks corresponding to low charge states are observed. We were unable to produce higher $\mathrm{m} / \mathrm{z}$ species, but we anticipate that the funnel will transmit ions up to the practical upper $\mathrm{m} / \mathrm{z}$ limit of the TOF-MS. In Figure 6c, the results for electrospraying a solution of serine are given. Many weakly bound noncovalent clusters are observed, indicating that heating does not occur as ions are transmitted through the funnel. In fact, under even more gentle conditions (see inset of Figure 6c) the octamer is almost exclusively observed. Previous results for serine clusters obtained with an ion funnel were substantially different and indicative of collisional heating [13].

\section{Conclusions}

Experimental and theoretical results for a modified ion funnel are presented. The implementation of greater spacing between the ring electrodes leads to enhanced ion compression and simplification of the manufacturing and assembly of the funnel. Furthermore, the funnel does not suffer from $\mathrm{m} / \mathrm{z}$ discrimination effects, ions are not heated unless heating is desired, and ions with varying masses including large masses are transmitted efficiently. A simple relationship for designing ion funnels or other ring electrodes is given and found to be in excellent agreement with more complex simulations. A new method for simulating ion trajectories at nonzero pressures is described. This method relies on ion mobility and explicit ion diffusion to simulate the motion of ions. These simulations were used to successfully design the modified ion funnel tested here.

\section{References}

1. Fenn, J. B.; Mann, M.; Meng, C. K.; Wong, S. F.; Whitehouse, C. M. Electrospray Ionization for Mass-Spectrometry of Large Biomolecules. Science 1989, 246, 64-71.
2. (a) Shaffer, S. A.; Tang, K.; Anderson, G. A.; Prior, D. C.; Udseth, H. R.; Smith, R. D. A Novel Ion Funnel for Focusing Ions at Elevated Pressure Using Electrospray Ionization Mass Spectrometry. Rapid Commun. Mass Spectrom. 1997, 11, 18131817; (b) Shaffer, S. A.; Prior, D. C.; Anderson, G. A.; Udseth, H. R.; Smith, R. D. An Ion Funnel Interface for Improved Ion Focusing and Sensitivity Using Electrospray Ionization Mass Spectrometry. Anal. Chem. 1998, 70, 4111-4119.

3. Shaffer, S. A.; Tolmachev, A.; Prior, D. C.; Anderson, G. A.; Udseth, H. R.; Smith, R. D. Characterization of an Improved Electrodynamic Ion Funnel Interface for Electrospray Ionization Mass Spectrometry. Anal. Chem. 1999, 71, 2957-2964.

4. (a) Lynn, E. C.; Chung, M. -C.; Han, C. -C. Characterizing the Transmission Properties of an Ion Funnel. Rapid Commun. Mass Spectrom. 2000, 14, 2129-2134. (b) Tolmachev, A.; Taeman, K.; Udseth, H. R.; Smith, R. D.; Bailey, T. H.; Futrell, J. H. Simulation-Based Optimization of the Electrodynamic Ion Funnel for High Sensitivity Electrospray Ionization Mass Spectrometry. Int. J. Mass Spectrom. 2000, 203, 31-47.

5. Taeman, K.; Tolmachev, A.; Harkewicz, R.; Prior, D. C.; Anderson, G. A.; Udseth, H. R.; Smith, R. D.; Bailey, T. H.; Rakov, S.; Futrell, J. H. Design and Implementation of a New Electrodynamic Ion Funnel. Anal. Chem. 2000, 72, 2247-2255.

6. (a) Wyttenbach, T.; Kemper, P. R.; Bowers, M. T. Design of a New Electrospray Ion Mobility Mass Spectrometer. Int. J. Mass Spectrom. 2001, 212, 13-23; (b) Seymour, J. L.; Syrstad, E. A.; Langley, C. C.; Turecek, F. Neutralization-Reionization of Ions Produced by Electrospray Instrument Design and Initial Data. Int. J. Mass Spectrom. 2003, 228, 687-702.

7. Taeman, K.; Tang, K.; Udseth, H. R.; Smith, R. D. A Multicapillary Inlet Jet Disruption Electrodynamic Ion Funnel Interface for Improved Sensitivity Using Atmospheric Pressure Ion Sources. Anal. Chem. 2001, 73, 4162-4170.

8. Tang. K. Q.; Tolmachev, A. V.; Nikolaev, E.; Zhang, R.; Belov, M. E.; Udseth, H. R.; Smith, R. D. Independent Control of Ion Transmission in a Jet Disrupter Dual-Channel Ion Funnel Electrospray Ionization. Anal. Chem. 2002, 74, 5431-5437.

9. Dahl, D. A. SIMION 3D (Version 7.0) Idaho National Engineering and Environmental Laboratory; Idaho Falls, ID, 2000.

10. O'Connor, P. B.; Costello, C. E.; Earle, W. E. A High Voltage RF Oscillator for Driving Multipole Ion Guides. J. Am. Soc. Mass Spectrom. 2002, 13, 1370-1375.

11. Mason, E. A.; McDaniel, E. W. Tansport Properties of Ions in Gases; Wiley: New York, 1988.

12. Gerlich, D. Applications of rf Fields and Collision Dynamics in Atomic Mass Spectrometry. J. Anal. At. Spectrom. 2004, 19, 581-590.

13. Wytennbach, T.; Bowers, M. T. Gas-Phase Conformations: The Ion Mobility/Ion Chromatography Method. Top. Curr. Chem. 2003, 225, 207-232. 